\title{
The high performance physician program: the impact of performance psychology in an otolaryngology department
}

\begin{abstract}
Objectives: To implement and evaluate the efficacy of a High Performance Physician Program (HPPP) in an otolaryngology department.

Design: This was a longitudinal cohort study, using surveys before and after the HPPP.

Methods: The HPPP involves individual assessment, reading, seminars, group discussion, and individual coaching. The program was administered by two performance psychologists who have worked extensively with Olympic and professional athletes and medical professionals. The HPPP consists of 4 sessions in a 4 -month period. It was administered separately for residents $(\mathrm{n}=11)$ and attendings $(\mathrm{n}=16)$.
\end{abstract}

Main outcome measures: The main outcome measure was burnout, as measured by the Maslach Burnout Inventory, delivered before and after the HPPP. We also considered participant testimonials as a qualitative outcome measure.

Results: Although the HPPP did not result in statistically significant changes we detected a surprisingly high degree of burnout and believe that it may go unnoticed. Subjectively, it was our experience that implementing a performance psychology program may help to decrease burnout.

Conclusion:Our study suggests that burnout can be a larger problem than expected, even when programs seem to be running smoothly. It is important to maintain awareness for possible adverse effects of burnout in resident training programs.

Keywords: burnout, maslach burnout inventory, physician wellness, performance psychology
Volume 7 Issue 2 - 2017

Darnbrough AL, Blakley BW, Osler FG
Department of Otolaryngology Health Sciences Centre University of Manitoba, Canada

Correspondence: Andrea Darnbrough Department of Otolaryngology Health Sciences Center University of Manitoba 820 Sherbrook St Winnipeg MB R3A IR9, Canada, Tel 204-7877773,Email andrea.darnbrough@gmail.com

Received: September 06, 2016 | Published: May 08, 2017
Abbrevaitions: HPPP, high performance physician program; TAIS, test of attentional and interpersonal style; MBI-HSS, maslach burnout inventory - human services survey;

\section{Introduction}

There is substantial literature documenting high levels of burnout, emotional exhaustion and depersonalization among both residents and attending physicians. ${ }^{1-12,13-17}$ Burnout is defined as "a pathological syndrome in which emotional depletion and maladaptive detachment develop in response to prolonged occupational stress". ${ }^{1}$ In Alberta, $48.6 \%$ of Canadian physicians have advanced burnout. ${ }^{2}$ Clinical practice, productivity and overall wellbeing are directly impaired as a result of moderate to high burnout levels. ${ }^{1-4}$ High levels of burnout have been correlated with increased medical error and decreased quality of patient care. ${ }^{5,6}$ Resident burnout may cause a decline in personal motivation and a decreased interest in job involvement leading to a decreased quality of residents' education..$^{7-9}$ The stresses of residency education, including intense work demands, sleep deprivation, lack of leisure time, and high degree of work-home interference are often sited as explanations for the high prevalence of burnout in medical residents. ${ }^{1,10,11}$

Significant burnout has been documented in both residents and attending otolaryngologists. Using the Maslach Burnout Inventory, moderate to high levels of burnout have been reported by $70 \%$ of American academic otolaryngologists, and $86 \%$ of American otolaryngology residents. ${ }^{12-14}$ To date, there are no studies of burnout in practicing Canadian Otolaryngologists.
The purpose of this study was to assess baseline levels of burnout for both residents and attendings in a Canadian Otolaryngology department, and evaluate the effects of a performance psychology program designed to address resident and physician wellness. We hypothesized that providing participants with an understanding of performance psychology would lessen burnout, as measured by the Maslach Burnout Inventory.

\section{Methods}

\section{Study design and participants}

The study design was a longitudinal cohort study, using surveys before and after the administration of the High-Performance Physician Program. Participants included the residents and attendings of the Department of Otolaryngology at the University of Manitoba, (residents $\mathrm{n}=11$, attendings $\mathrm{n}=16$ ).

\section{The high performance physician program (HPPP)}

The program was created and administered by two performance psychologists with extensive experience with both high performance athletes and medical personnel. The program consisted of four 3-hour sessions over a 4-month period, which included individual assessment, reading, seminars, group discussion, and individual coaching. Core principles included in our intervention were: perspective, self-assessment, imagery and rehearsal, the importance of recovery, emotional preparation and team building (see Appendix 1 for a summary of the HPP curriculum). Each participant was also evaluated using the Test of Attentional and Interpersonal Style (TAIS) testing at the 
onset of the program to individualize learning. A debriefing session for residents and attendings was held at the conclusion of the program.

\section{Survey administration}

Participants were contacted by email to complete a web-based survey 1 week before and 1 month after the HPPP; responses were anonymous and voluntary. The survey utilized was the Maslach Burnout Inventory - Human Services Survey (MBI-HSS). ${ }^{12}$

\section{Survey scoring - MBI-HSS}

This 22-item questionnaire evaluates 3 domains of burnout: personal accomplishment (8 questions) emotional exhaustion (9 questions) and depersonalization (5 questions). Participants responded to each question using a 7-point Likert scale from 'never' $(0)$ to 'everyday' (6). According to a previously published study on 1104 medical personnel, a high degree of burnout is defined as a combination of low personal accomplishment, and high emotional exhaustion and depersonalization. Physicians are more likely than other groups to score high on the personal accomplishment; therefore moderate burnout has been defined as moderate to high scores for emotional exhaustion and depersonalization, with high personal accomplishment. ${ }^{13-15}$ Since burnout is a multidimensional construct, the three subscale scores are not combined; personal accomplishment, emotional exhaustion and depersonalization are considered separately. ${ }^{12}$ Emotional exhaustion is considered the most relevant parameter in physicians. ${ }^{12}$

\section{Statistical analysis}

Data departures from the normal distribution were found for some variables so the Mann-Whitney-U test was applied using IBM (SPSS 19.0 software). The significance level was 0.05 . In addition, the numbers of residents and attending physicians were categorized into LOW, MODERATE and HIGH for Personal Accomplishment, Emotional Exhaustion and Depersonalization as recommended by MBS-HSS. ${ }^{12}$

\section{Results}

Our response rates were $77 \%$ for residents and $50 \%$ for attending otolaryngologists.

All participants had high levels of Personal Accomplishment, which is consistent with previously published literature on medical professionals. ${ }^{13-15}$ The changes in the sum of scores in each of the other two domains, Emotional Exhaustion, and Depersonalization before and after participation in the HPPP were not statistically significant (Table 1).

Table I Table of p-values (2-tailed, Mann-Whitney $U$ test) for differences in the ranks of the levels (Low, Moderate, or High) of responses in each domain for pre- versus post- participation and for residents versus attendings

\begin{tabular}{lll}
\hline & Pre vs. Post & Residents vs.Attendings \\
\hline Personal Accomplishment & $\mathrm{I}$ & $\mathrm{I}$ \\
Emotional Exhaustion & 0.397 & $0.70 \mathrm{I}$ \\
Depersonalization & 0.088 & 0.656 \\
\hline
\end{tabular}

Power analysis ( $80 \%$ at the $\mathrm{p}=0.05$ level) was conducted assuming a normal distribution for pre- and post-participation and for residents versus attendings for all 22 variables indicated maximum observed power of $20 \%$.

\section{Residents}

All residents scored high on Personal Accomplishment both before and after the HPPP.
Before completing the HPPP, for Emotional Exhaustion: three of ten residents had high scores, three of ten had moderate scores, and four of ten had low scores. After the HPPP, for Emotional Exhaustion: one of seven residents scored high, three of seven scored moderate, and three of seven scored low. These results are summarized in Figure 1.

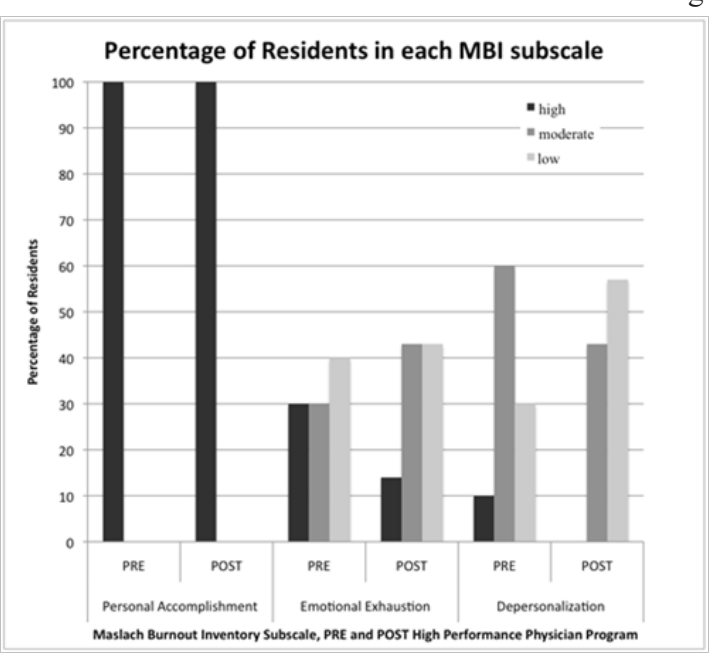

Figure I Percentage of residents in each MBI subscale. The percentage of residents within high, moderate, or low categories for each subscale of the Maslach Burnout Inventory (MBI) before and after the High Performance Physician Program.

\section{Attending otolaryngologists}

All attendings scored high on Personal Accomplishment both before and after the HPPP. Before completing the HPPP, for Emotional Exhaustion: four of eight attendings scored high, one of eight scored moderate, and three of eight scored low. After completing the HPPP, for Emotional Exhaustion: one of eight attendings scored high, four of eight scored moderate, and three of eight score low. These results are summarized in Figure 2 .

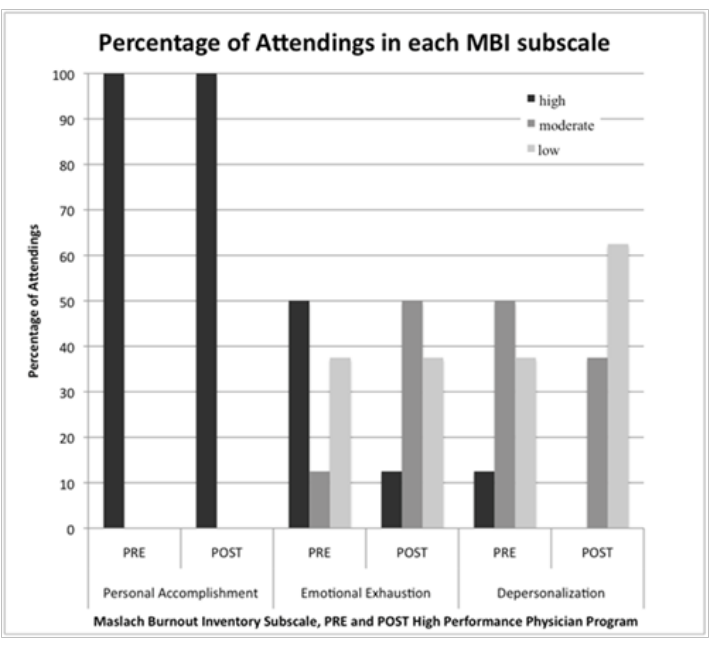

Figure 2 Percentage of attendings in each MBI subscale. The percentage of attendings within high, moderate or low categories for each subscale of the Maslach Burnout Inventory (MBI) before and after the High Performance Physician Program.

\section{Burnout}

Since all residents and attendings scored high on Personal Accomplishment, none of the participants met the criteria for high levels of 
burnout. Prior to the HPPP, five of ten residents scored either moderate or high on both Emotional Exhaustion and Depersonalization, suggesting that $50 \%$ of residents showed moderate levels of burnout. After the HPPP, $29 \%$ of residents showed moderate levels of burnout. Prior to the HPPP, 50\% (4/8) of attendings showed moderate levels of burnout. After the HPPP, 37.5\% (3/8) of attendings showed moderate levels of burnout. These results are summarized in Figure 3.

The data trends are shown in graphic form in Figure 4.

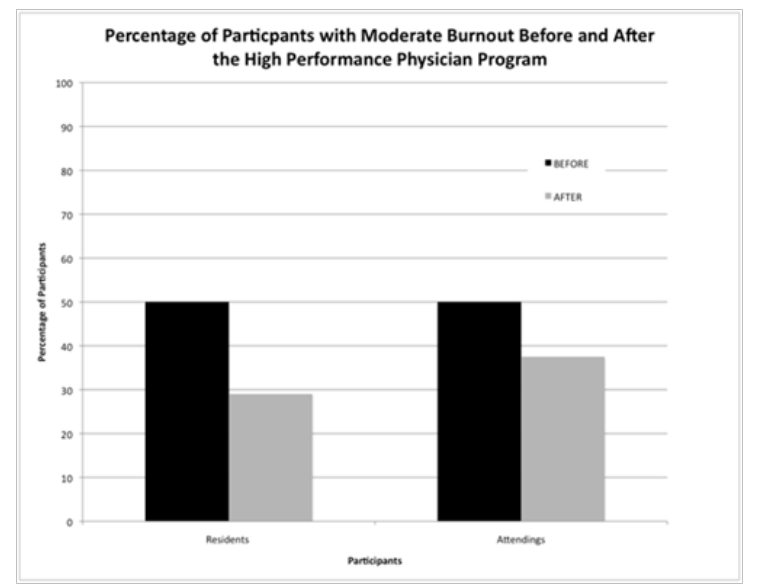

Figure 3 Percentage of Participants with Moderate Burnout before and After the High Performance Physician Program (HPPP). Prior to the HPPP, $50 \%$ of residents and $50 \%$ of attendings met criteria for moderate levels of burnout. After the HPPP, $29 \%$ of residents and $37.5 \%$ of attendings met the criteria for moderate levels of burnout.

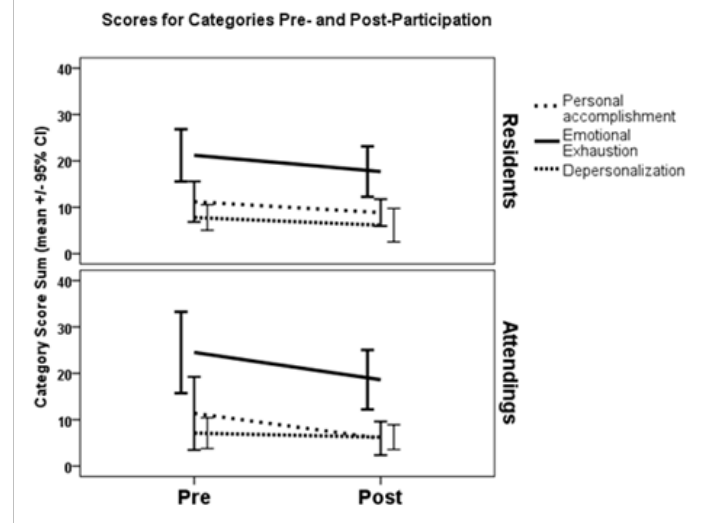

Figure 4 Changes in the Sums of Scores for Personal Accomplishment, Emotional Exhaustion and Depersonalization. The down-sloping lines are suggestive of improvement in these three scores but the changes were not statistically significant.

\section{Discussion}

This study demonstrated that moderate burnout is prevalent among both residents and attendings within a Canadian Otolaryngology department; this is consistent with the American findings. ${ }^{13,14}$ Existing literature on burnout has consistently demonstrated negative implications for the learning environment, patient care, the quality of residency training, and overall physician wellness. ${ }^{1,4,9}$ Despite its harmful effects, burnout remains a disturbingly under recognized condition among physicians. ${ }^{14}$
Although the Maslach Burnout Inventory clearly demonstrated burnout in both residents and attendings, we suspect that the factors contributing to burnout, as well as ability to manage these factors, are different for the two groups given that each group is at a different career stage. Although both groups experience intense work demands, sleep deprivation, lack of leisure time, and a high degree of work-home interference, there are also notable differences. Many residents are reported to acknowledge they had "altruistic ideals, fantasies of healing the sick and heroic images of themselves" when they entered medicine. ${ }^{16}$ In attending otolaryngologists, burnout may be more related to inadequate resources to do the job properly, time pressure during office visits, and lack of control over scheduling; as well as a variety of other personal and professional factors. ${ }^{17,18}$ We did not seek to understand these differences in the current study, however it is important to realize that residents and attendings may be two very different groups when interpreting our results.

To our knowledge, this is the first study to implement a performance psychology program in a surgical department. The feedback from the program was generally positive, particularly from the resident group. Resident testimonials included: "Great experience: helped with perspective, dealing with difficult personalities and finding ways to de-stress and refocus"; "I have learned that it is essential to stop and have time to recover so that the ensuing day/week/residency is not insurmountable" and "I realized I was probably working somewhere at $60-70 \%$ of capacity and am working towards $100 \%$ after these few months I am probably at $80-85 \%$ already". Of note, there were no negative comments from residents. Attending testimonials were mixed in nature, and included: "I feel more positive attitudes at work and in life in general"; "The HPPP was helpful in gaining perspectives, and to provide concepts for stress management"; "I don't think the HPPP did anything"; and "I felt the program served no purpose and lacked direction". The number of positive and negative testimonials from attendings were approximately equal. Residents may have benefited more from this intervention than attendings; perhaps the needs of attending otolaryngologists were not adequately addressed by our program.

We believe that our small sample size resulted in statistically non-significant results, however our small sample size also included most of the department. Our results demonstrated that the total number of participants that met the criteria for burnout decreased in both the resident and attending groups. Prior to the HPPP, $50 \%$ of residents and $50 \%$ of attendings met criteria for moderate levels of burnout. After the HPPP, $29 \%$ of residents and $37.5 \%$ of attendings met the criteria for moderate levels of burnout (Figure 3). It is also important to note that the data for all three parameters in the MBI (Personal Accomplishment, Emotional Exhaustion and Depersonalization) trended in the direction suggestive of decreased burnout (Figure 4). Subjectively, our experience has been an improvement in both individual functioning and in the overall working environment. Even if only a few individuals were to experience less burnout, we feel there is value in providing physicians with resources, such as a performance psychology program, to lessen burnout.

Our small sample size was a major limitation of this study. Participation in this study was voluntary and required a significant 'after-hours' time commitment from already busy practicing physicians; we felt our participation rate was satisfactory given the circumstances. Other potential limitations include recall bias due to inaccurate self-reporting and response bias attributable to non-responders.

Recall bias may also have contributed to our non-significant results. It is possible that our pre-HPPP burnout measure is actually an 
underrepresentation of the true level of burnout that was present. It has been suggested that there is an impact of 'the culture of medicine', which promotes perfectionism, self-sacrifice, and 'workaholic standards' ${ }^{19}$ Physicians may experience "a perceived pressure NOT to show weakness", which could potentially cause inaccurate self-reporting that differs between the pre and post HPPP state..$^{19}$ If pre-HPPP burnout was underreported, it would be difficult to show significant improvement; it is unclear whether recall bias impacted our study in this way.

It is important to note the HPPP was not designed specifically to decrease burnout rates in our department, and the psychologists administering the program were not aware that MBI would be utilized to measure the effects of the HPP program. The HPPP was designed to improve performance in all domains of a surgical department. A more robust effect of the HPPP might be measured in the fields of group dynamics, occupational stress, interpersonal relationships or even surgical performance; however these parameters are difficult to measure objectively. Future research in this area is required.

It is important that both residents and attendings have the necessary psychological tools to prevent daily stressors from leading to burnout which could have serious implications for patient care, resident learning and overall physician wellness. Based on participant testimonials about HPPP, performance psychology may be beneficial. Given our objective findings and the significant limitations of the current study discussed in the preceding paragraphs, it is unclear whether the HPPP is an effective at preventing burnout.

\section{Conclusion}

Psychological stress and burnout are problems that are often present in spite of a superficial view to the contrary. Despite the positive subjective experience of participants, the objective results did not demonstrate a measurable difference in burnout before and after the program. Emphasis should be placed on further research to develop effective burnout prevention strategies for both residents and attendings.

\section{Acknowledgements}

We would like to thank Cal Botterill, Jason Brooks and Aman Hussain for creating and administering the High Performance Physician Program.

\section{Conflicts of interest}

Author declares there are no conflicts of interest.

\section{Funding}

None.

\section{References}

1. Thomas NK. Resident Burnout. JAMA. 2004;292(23):2880-2889.
2. Boudreau RA, Grieco RL, Cahoon SL. The pandemic from within: two surveys of physicians' burnout in Canada. Can J Commun Mental Health. 2006;25(2):71-88.

3. Clever LH. Who is sicker: Patients or Residents? Residents' distress and the care of patients. Ann Int Med. 2002;136(5):391-393.

4. CollierVU, McCue JD, MarkusA, et al. Stress in medical residency: Status quo after a decade of reform? Ann Intern Med. 2002;136(5):384-390.

5. Spickard A, Gabbe SG, Christensen JF. Mid-career burnout in generalist and specialist physicians. JAMA. 2002;288(12):1447-1450.

6. Shanafelt TD, Bradley KA, Wipf JE, et al. Burnout and self-reported patient care in internal medicine residency program. Ann Intern Med. 2002;136(5):358-367.

7. Fahrenkopf AM, Sectish TC, Barger LK, et al. Rates of medication errors among depressed and burnout residents: perspective cohort study. BMJ. 2007;336(7642):488-491.

8. Colford JM, McPhee SJ. The raveled sleeve of care: managing the stress of residency training. JAMA. 1989;261(6):889-893.

9. Hsu K, Marshall V. Prevalence of depression and distress in a large sample of Canadian residents, interns and fellows. Am $J$ Psych. 1997;144(12):1561-1566.

10. Vu TT, Nguyen LH. Residents' satisfaction with Canadian otolaryngology-head and neck surgery programs. J Otolaryngol Head Neck Surg. 2010;39(2):207-213.

11. Gaba DM, Howard SK. Fatigue among clinicians and the safety of patients. $N$ Eng J Med. 2002;347(16):1249-1255.

12. Maslach C, Jackson SE, Leiter MP.Maslach Burnout Inventory Manual. ( $3^{\text {rd }}$ edn). Consulting Psychologists Press, Palo Alto, CA, USA. 1996.

13. Golub JS, Johns MM, Weiss PS, et al. Burnout in academic faculty of otolaryngology-head and neck surgery. Laryngoscope. 2008;118(11):1951-1956.

14. Golub JS, Weiss PS, Ramesh AK, et al. Burnout in Residents of Otolaryngology-head and neck surgery: A national inquiry into the health of residency training. Acad Med. 2007;82(6):596-601.

15. Lee RT, Ashforth BE. A metanalytic examination of the three dimensions of job burnout. J Appl Psych. 1996;81(2):123-133.

16. Willcock SM, Daly MG, Tennant CC, et al. Burnout and psychiatric morbidity in new medical graduates. Med J Aust. 2004;181(7):357-360.

17. Linzer M, Visser MR, Oort FJ, et al. Predicting and preventing physician burnout: results from the United States and the Netherlands. Am J Med. 2001;111(2):170-175.

18. Fletcher AM, Pagedar N, Smith RJ. Factors Correlating with Burnout in Practicing Otolaryngologists. Otolaryngol Head Neck Surg. 2012;146(2):234-239.

19. Miller MN, McGowen KR. The Painful Truth: Physicians are not invincible. South Med J. 2000;93(10):966-973. 